\title{
Microvascular Rarefaction and Myocardial Fibrosis in Hypertrophic Obstructive Cardiomyopathy: A Histopathological Comparison of Pediatric and Adult Patients
}

\author{
Mingkui Zhang, MD, ${ }^{1}$ Zhengjie Zhang, MD,${ }^{1}$ Hui Xue, $M D,{ }^{1}$ Lixin Fan, $M D,{ }^{1}$ Yan Weng, $\mathrm{MD}^{2}$ \\ ${ }^{1}$ Heart Center, First Hospital of Tsinghua University, Beijing China; \\ ${ }^{2}$ The Department of Pathology, First Hospital of Tsinghua University, Beijing China
}

\section{ABSTRACT}

Background: Hypertrophic obstructive cardiomyopathy (HOCM) is a genetic cardiomyopathy characterized by microvascular ischemia and myocardial fibrosis. Microvessels play an important role in myocardial fibrosis in HOCM. However, the changes of myocardial microvessels and myocardial fibrosis in pediatric and adult patients with HOCM remain unclear. This study was to investigate the changes in myocardial microvessel density (MVD) and myocardial fibrosis in pediatric and adult patients with HOCM.

Methods: We analyzed the changes in MVD and myocardial fibrosis in myectomy left ventricular (LV) septal wall specimens in 12 adult patients and five pediatric patients with HOCM. Control myocardium from the LV septal wall was collected at autopsy of 5 adults and 4 pediatric individuals, who died of non-cardiac causes.

Results: There was no significant difference in MVD between pediatric $H O C M$ patients and control subjects $(706.4 \pm 187.5$ vs. $940.2 \pm 491.1, \mathrm{P}>0.05$ ), but the myocardial fibrosis area ratio was significantly increased in HOCM than in control subjects $(10.6 \pm 3.5$ vs. $4.9 \pm 1.2, \mathrm{P}<0.01)$. MVD was significantly reduced, and myocardial fibrosis area ratio was significantly higher in adult HOCM patients than in control subjects (i.e. $523.3 \pm 209.4$ vs. $845.7 \pm 260.7, \mathrm{P}<0.05 ; 12.8 \pm 5.1$ vs. $4.4 \pm 1.3, \mathrm{P}<0.05)$. There was no significant difference in MVD and myocardial fibrosis between pediatric and adult HOCM patients $(706.4 \pm 187.5$ vs. $523.3 \pm 209.4, \mathrm{P}>0.05 ; 10.6 \pm 3.5$ vs. $12.8 \pm 5.1, \mathrm{P}>0.05)$.

Conclusions: Pediatric and adult patients with HOCM have high myocardial fibrosis. The present findings suggest that myocardial microvascular density lesions contribute to myocardial fibrosis during childhood.

\section{INTRODUCTION}

Hypertrophic obstructive cardiomyopathy (HOCM) is a hereditary cardiomyopathy characterized by asymmetric

Received August 30,2021; received in revised form November 24, 2021; accepted November 28, 2021.

Correspondence: Mingkui Zhang, No 6. 1st street, Fiuxianqiao, Chaoyang District, 100016, Beijing, China, Telephone 86-10-64308006 (e-mail: mingkuizhang@163.com). hypertrophy of the myocardium, disarray myocardial cells, and myocardial fibrosis. The prevalence of hypertrophic cardiomyopathy is about $0.2 \%$ in the population [Maron 2018]. The clinical manifestations of hypertrophic cardiomyopathy vary widely. Many patients can have no clinical symptoms, and some patients have angina pectoris symptoms due to myocardial hypertrophy, insufficient energy supply, or ischemia caused by myocardial bridges. Syncope easily occurs with left ventricular outflow tract obstruction; some patients have sudden cardiogenic death as the first symptom [Antunes 2020]. Cohort studies have found an annual mortality rate of $1 \%$, with some patients progressing to heart failure or atrial fibrillation, and an increased risk of secondary embolism [Weissler-Snir 2019].

The characteristic manifestations of hypertrophic cardiomyopathy are left ventricular hypertrophy, myocardial fibrosis, and microvascular ischemia [Aguiar Rosa 2021]. The imbalance between myocardial energy expenditure and energy supply caused by alterations in the coronary vascular system is the mechanism of the clinical symptoms of hypertrophic cardiomyopathy. Myocardial capillary density is about $3000-4000 / \mathrm{mm}^{3}$ under normal conditions, and blood flow increases sixfold in emergencies situations to accommodate energy demands [Marszalek 2019]. A study of postoperative myocardial tissue in patients with hypertrophic cardiomyopathy revealed a significant decrease in cardiac capillary density, accompanied by an increase in the area of myocardial fibrosis [Tian 2018]. But it is unclear, however, whether and to what extent pathological diffuse fibrosis occurs in childhood HCOM. The purpose of this study was to determine whether diffuse myocardial fibrosis and reduced MVD occur in pediatric and adult patients and further understand the development and evolutionary mechanisms of hypertrophic cardiomyopathy.

\section{METHODS}

Study population: We studied the pediatric and adult patients with HOCM who underwent transaortic extended septal myotomy (Morrow operation) at the Heart Center of the First Affiliated Hospital of Tsinghua University from March 2004 to December 2018. Control myocardium from the LV septal wall was collected at autopsy of adult and pediatric individuals, who died of non-cardiac causes. Hypertrophic cardiomyopathy was diagnosed as left ventricular 
thickness or septal thickness $\geq 15 \mathrm{~mm}$, or left ventricular outflow tract pressure difference $>30 \mathrm{mmHg}$ at rest, or $>50 \mathrm{mmHg}$ at provocation, without other factors causing myocardial hypertrophy. In the case of children, increased $L V$ wall thickness is defined as wall thickness $\geq 2$ standard deviations above the mean (z score $\geq 2$ ) for age, sex, or body size [Gersh 2011; Elliott 2014]. Clinical information was obtained from the hospital's electronic medical record system, including medical history, physical examination, electrocardiogram, 24-hour ambulatory electrocardiogram, echocardiogram, coronary angiography, and myocardial magnetic resonance examination. Coronary artery disease ( $>50 \%$ stenosis on coronary angiography), uncontrollable hypertension (defined as blood pressure above 140/90 $\mathrm{mmHg}$ ), and renal dysfunction were excluded. The study was approved by the Ethics Committee of the First Affiliated Hospital of Tsinghua University, and written informed consent was obtained from the participants or their parents or legal guardians.

Echocardiography: Standard transthoracic M-mode, 2-dimensional, pulsed, or continuous wave Doppler images were obtained with an IE33 Color Doppler Ultrasound System (Philips Healthcare, Andover, MA, USA). Parameter acquisition was performed, according to the American Society of Echocardiography guidelines. The detection of peak velocity and differential pressure across the left ventricular outflow tract was calculated using the simplified Bernoulli equation. Left ventricular outflow tract obstruction was defined as hypertrophic cardiomyopathy diagnosed as left ventricular thickness or septal thickness $\geq 15 \mathrm{~mm}$ or left ventricular outflow tract differential pressure $>30 \mathrm{mmHg}$ at rest or $>50 \mathrm{mmHg}$ at provocation.

Surgical technique: A modified Morrow procedure is used, and intraoperative transesophageal ultrasonography routinely was applied. After median sternotomy, the pressure gradient between the left ventricle to the ascending aorta was measured directly. Extracorporeal circulation was established via aortic and right atrial cannulation, cold blood hyperkalemic cardioplegia was infused into the aortic root after the distal ascending aorta was clamped. A transverse aortotomy toward the mid-noncoronary sinus and aortic annulus was performed. The continuous resection was commenced at the nadir of the right aortic sinus leftward toward the mitral valve annulus and apically to the base of the papillary muscles, involving the abnormal tendons and muscle bundles near the mitral papillary muscle. Transesophageal ultrasound was used after the patients were weaned from cardiopulmonary bypass. Individualized administration of $\beta$-blocker routinely was used during postoperative management.

Histological examination: Myocardial tissue samples were taken from patients with HOCM after septal myectomy. Control myocardium from the LV septal wall was collected at autopsy of individuals who died of noncardiac causes. The samples were fixed in $10 \%$ buffered formalin, embedded in paraffin, and cut into $4 \mu \mathrm{m}$ thick tissue sections for immunohistochemical staining. Dewaxing and hydration were performed by a series of applications of xylene and alcohol. Microwave antigen repair (0.1 M citrate buffer, $\mathrm{pH}$ 6.0) for $10 \mathrm{~min}$. Mouse anti-human monoclonal antibody CD31 (UMAB30, ZSGB$\mathrm{BIO}$, China) was incubated overnight at $4^{\circ} \mathrm{C}$, rinsed in buffer, incubated with rabbit anti-mouse (IgG, PV-6000, ZSGB-BIO, China) for $30 \mathrm{~min}$ at room temperature, and DAB (OriGene Technologies, China) stained for $30 \mathrm{~min}$. Finally, after incubation with diaminobenzidine for $30 \mathrm{~min}$ at room temperature, the slides revealed immunoreactivity for CD31.

MVD evaluation: CD31 immunohistochemically stained images were acquired by applying light microscopy, and the images were magnified 200 times with brown staining of vascular endothelial cells. Microvasculature included capillaries (area $10-78.5 \mu \mathrm{m}^{2}$, lumen diameter $<10 \mu \mathrm{m}$ ) and precapillary arteries (area 78.5-314 $\mu^{2}$, lumen diameter $<10-20 \mu \mathrm{m}$ ), microvessels were tagged by the pathologists on the monitor image and counted in 6 fields at $\times 200$ magnification using Image-Pro Plus 6.0 image analysis software (Media Cybernetics Inc., Buckinghamshire, UK) to calculate microvessels. Finally, the mean number of microvessels per square millimeter was calculated and expressed as the MVD [Tian 2018].

Masson staining and determination of myocardial fibrosis: Paraffin sections were dewaxed with xylol and soaked into a series of a gradient concentrations from 99 to $95 \%$ of alcohol, and distilled water washing in turn. The slices were put in a solution of hematoxylin for $3 \mathrm{~min}$. Subsequently, a color change was carried out with lithium carbonate. Slices were washed in pure water and colored with red Ponceau staining (oven at $30^{\circ} \mathrm{C}$ for $20 \mathrm{~s}$ at $45 \mathrm{~kW}$ ). The slice was put in acid water and phosphomolybdic acid $(1 \mathrm{~min})$, and then aniline blue was added and washed with acid water. Six fields of view randomly were selected at $\times 200$ magnification, and the percentage $(\%)$ of the area of fibrosis was calculated after labeling by a pathologist using Image-Pro Plus 6.0 image analysis software (Media Cybernetics Inc., Buckinghamshire, UK) [Tian 2018].

Statistical methods: Statistical data were analyzed using SPSS 22.0 (SPSS Inc., Chicago, IL, USA). Data were expressed as mean \pm standard deviation. Continuous variables were compared between the two groups using an independent t-test, while categorical variables were compared using the chi-square test. $\mathrm{P}$ values less than 0.05 were used as a test for significant differences.

\section{RESULTS}

Patient characteristics: In this study, there were 12 adult patients and five pediatric patients with HOCM. Control myocardium from the LV septal wall was collected at autopsy of four adults and five pediatric individuals who died of noncardiac causes. Clinical data and echocardiographic parameters are shown in Table 1 . There were no significant differences in age or gender between adult patients with HOCM and control subjects, and with the same results between pediatric patients and control subjects.

Microvessel density and myocardial fibrosis: As shown in Figure 1, both pathologists showed agreement with respect to MVD, myocardial fibrosis in the myocardium of pediatric, adult patients, and control subjects, respectively. (Figure 1) 
Table 1. Summary of clinical and echocardiographic data

\begin{tabular}{|c|c|c|c|c|}
\hline Ages, years & $9.3 \pm 4.0$ & $5.2 \pm 1.7$ & $47.4 \pm 7.9$ & $41.5 \pm 15.4$ \\
\hline Symptom, n (\%) & $1(20 \%)$ & - & $11(92 \%)$ & - \\
\hline ECG ST-T changes, n (\%) & $2(40 \%)$ & - & $7(58 \%)$ & - \\
\hline Systolic anterior motion & $5(100 \%)$ & - & $10(83 \%)$ & - \\
\hline LVOT PG at rest, $\mathrm{mm} \mathrm{Hg}$ & $89.4 \pm 29.4$ & - & $96.8 \pm 44.1$ & - \\
\hline
\end{tabular}

ECG, electrocardiograph; LVOT PG, left ventricular outflow tract pressure gradient

In Figure 2, MVD was not significantly different between pediatric patients with HOCM and controls (Figure 2A) $(706.4 \pm 187.5$ vs. $940.2 \pm 491.1, P>0.05)$; MVD was significantly lower in adult HOCM patients compared with controls (Figure $2 \mathrm{~B})(523.3 \pm 209.4$ vs. $845.7 \pm 260.7, P<0.05)$. (Figure 2) By contrast, the myocardial fibrosis ratio was significantly higher in pediatric HOCM patients than in the controls (Figure $2 \mathrm{C})(10.6 \pm 3.5$ vs. $4.9 \pm 1.2, P<0.01)$; meanwhile, it was significantly higher in adult HOCM patients than in the control group (Figure $2 \mathrm{D})(12.8 \pm 5.1$ vs. $4.4 \pm 1.3$, $P<0.05)$. There were no significant differences in MVD (Figure 2E) $(706.4 \pm 187.5$ vs. $523.3 \pm 209.4)$ and myocardial fibrosis (Figure $2 \mathrm{~F})(10.6 \pm 3.5$ vs. $12.8 \pm 5.1)$ between pediatric and adult HOCM patients $(P>0.05)$.

\section{DISCUSSION}

Our study provides evidence that both pediatric and adult HOCM patients had a higher myocardial fibrosis ratio (\% area), and adult HOCM patients had a lower MVD compared with controls. There were no significant differences in MVD and myocardial fibrosis in pediatric and adult HOCM patients. To our knowledge, this is the first report on the comparison of MVD and myocardial fibrosis in adult versus pediatric patients with HOCM.

We did not find literature reports on myocardial MVD changes in children with HOCM. However, it has been shown that mortality in adolescent patients with hypertrophic cardiomyopathy is significantly higher than in adult patients, and the peak age of death is between 9 and 12 years [Ostman-Smith 2008]. In the early stages of hypertrophic cardiomyopathy, there is coronary vascular endothelial cell dysfunction, vasoconstriction, decreased myocardial perfusion, and myocardial ischemia [Timmer 2013]. Myocardial ischemia caused by microvascular dysfunction is an important pathophysiologic component of hypertrophic cardiomyopathy, promoting myocardial fibrosis, adverse left ventricular remodeling, and impacting clinical course and outcome in HOCM patients [Aguiar Rosa 2021; Raman 2019]. Analysis of patients with childhood hypertrophic cardiomyopathy using single-photon emission computed tomography (SPECT) myocardial perfusion studies have shown that myocardial perfusion deficit, myocardial ischemia leads to clinical symptoms, and predicted sudden death and adverse cardiac events in children hypertrophic cardiomyopathy patients [Ziolkowska 2021]. Left ventricular outflow tract pressure gradients, increased vascular impedance, and increased vessel wall tension can all contribute to ventricular diastolic dysfunction and abnormal myocardial perfusion [Knaapen 2008; Güçlü 2015]. In this study, the left ventricular outflow tract pressure gradient was $89.4 \mathrm{mmHg}$ at rest in pediatric HOCM patients, but without a significant difference in MVD compared with controls. There were $40 \%$ pediatric HOCM patients with preoperative electrocardiograph (ECG) ST-segment changes, presumably suggesting that myocardial perfusion disturbance may be an early pathological change in childhood, and clinical symptoms may be related to coronary vascular endothelial dysfunction, reduced myocardial perfusion, and myocardial ischemia.

In this study, we demonstrated that MVD was significantly lower, and the myocardial fibrosis ratio was higher in adult patients with HOCM compared with controls. Postmortem examination of hypertrophic cardiomyopathy heart revealed increased myocardial fibrosis and reduced capillary density [Hughes 2004]. Our study is consistent with the findings of previous studies. In recent years, several authors have used myocardial perfusion magnetic resonance imaging (MRI) to evaluate ischemia in adult HOCM patients [Iles 2015; Moravsky 2013]. Matsunaka et al. [Matsunaka 2003] reported that first-pass MRI with delayed contrast-enhanced MRI is useful for evaluating the myocardial injury and for predicting left ventricular regional contractile function in patients with HOCM. MRI perfusion abnormalities at rest are correlated with myocardial fibrosis and risk factors for sudden cardiac death, and myocardial perfusion MRI scans are of great value in evaluating microvascular function [Chiribiri 2015]. Reduced myocardial perfusion and coronary flow reserve are the main factors contributing to myocardial ischemic symptoms and affecting long-term prognosis [Güçlü 2015]. We found a left ventricular outflow tract pressure gradient of $96.8 \mathrm{mmHg}$ and significantly reduced MVD in adult HOCM 

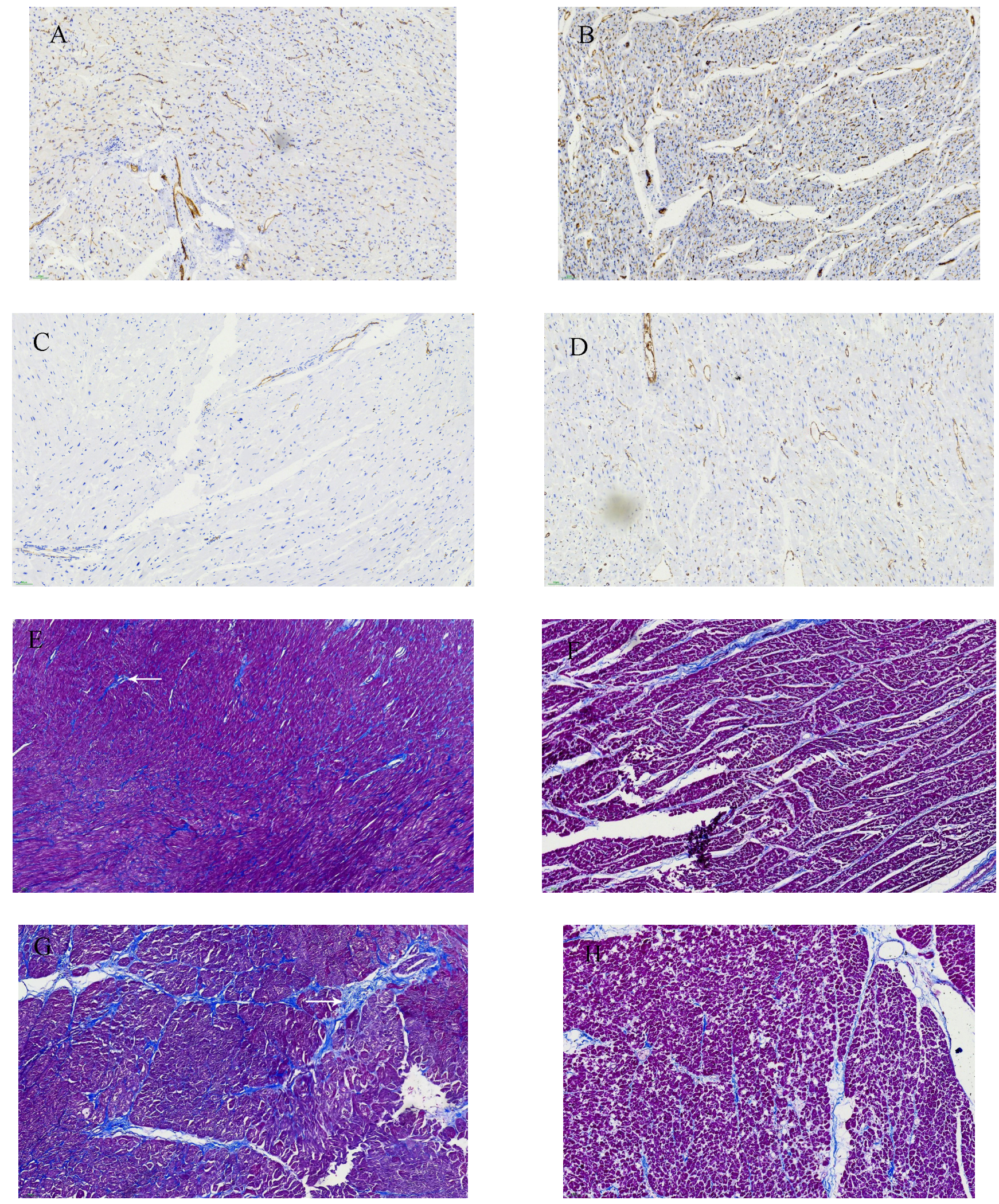

Figure 1. MVD and myocardial fibrosis in pediatric, adult HOCM patients compared with control subjects. Microvessels are stained in brown and myocardial fibrosis is stained in blue. The MVD in pediatric patients (A) and controls (B), adult patients (C), and subjects (D). Myocardial fibrosis area (\%) in pediatric patients $(E)$ and controls $(F)$, in adult patients $(G)$ and controls $(H)$. 
patients, and reduced coronary MVD was found in both autopsy and surgical tissue specimens from adult patients with HOCM. Left ventricular outflow tract pressure gradient has a decisive influence on coronary vascular remodeling and microvascular hypoperfusion, and reduced vascular density and reduced myocardial perfusion further affect the myocardial contractile function and lead to adverse cardiac outcomes. Meanwhile, cardiac vascular impedance can affect left ventricular wall pressure due to severe diastolic insufficiency, which causes increased left ventricular end-diastolic filling pressures [Knaapen 2008; Güçlü 2015].

Our study confirmed the finding of significant myocardial fibrosis in pediatric patients with HOCM, suggesting the early appearance of vascular endothelial cell lesions, reduced myocardial perfusion, myocardial interstitial remodeling, and microstructural fibrosis in pediatric patients. Fernlund and colleagues [Fernlund 2017] found that serum histone S, endothelial inhibitor, matrix metalloproteinase-9, vascular endothelial growth factor receptor, vascular and intercellular adhesion molecules were significantly elevated and negatively correlated with myocardial perfusion in pediatric patients with HOCM. Increased type I collagen synthesis early in hypertrophic cardiomyopathy was an early manifestation of myofilament gene mutations [Ho 2010]. Myocardial fibrosis is progressively aggravated, and abnormal myocardial energy utilization and perfusion deficits are responsible for myocardial fibrosis, myocardial fibrosis is associated with adverse myocardial remodeling and adverse cardiac events [Raman 2019]. Left ventricular outflow tract obstruction plays an important role in myocardial fibrosis and left ventricular diastolic dysfunction in patients with hypertrophic cardiomyopathy [Avegliano 2019]. Therefore, early outflow tract sparing surgery should be performed in patients with left ventricular outflow tract obstruction.

In this study, the main clinical symptoms of the patients were chest tightness, breath-holding, decreased activity tolerance, and arrhythmias, and seven adult patients (58\%) had ST-T changes on the ECG. Tangwiwat et al. [Tangwiwat 2019] suggested that ECG T-wave inversion was associated with apical hypertrophic cardiomyopathy, and fragmented QRS wave clusters were associated with myocardial fibrosis. Histological studies have found that pediatric patients are prone to sudden death and older patients are prone to heart failure [Galati 2016]. Myocardial fibrosis is a characteristic feature of hypertrophic cardiomyopathy and is a major cause of sudden death, ventricular arrhythmias, and cardiac failure. The present study showed five cases of ventricular arrhythmias and one case of atrial fibrillation in the present group of patients. But today, there is a lack of precise and valid risk stratification assessment measures [Ariga 2019].

A limitation of this study was its retrospective nature. While the sample size was small, a larger sample size could have further investigated the correlation between myocardial histological changes and left ventricular outflow tract pressure changes, as well as the correlation analysis between histological characters and postoperative long-term outcomes.

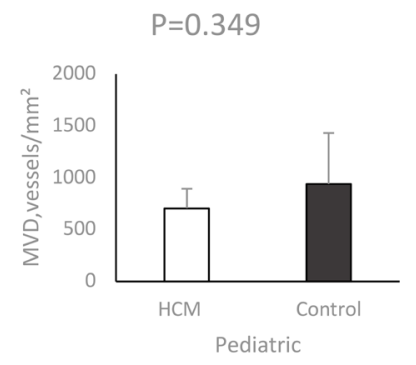

A

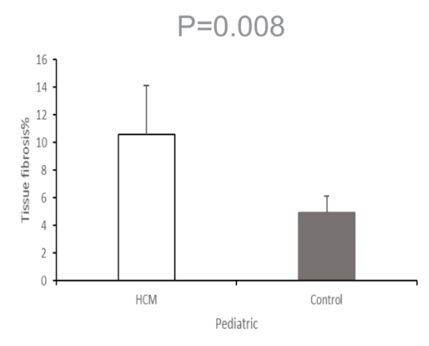

C

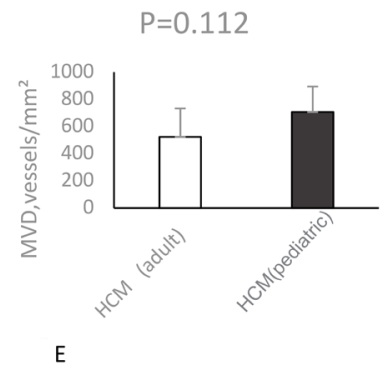

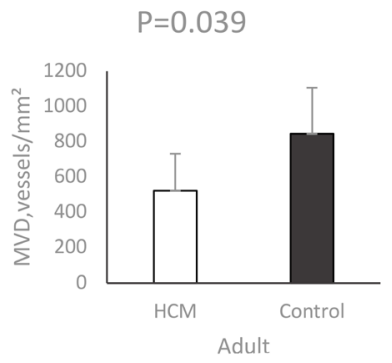

B

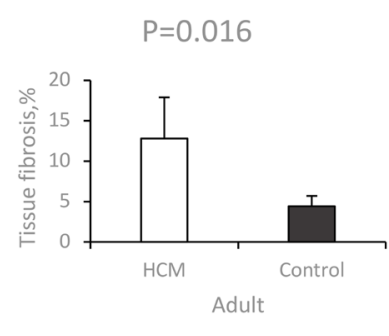

D

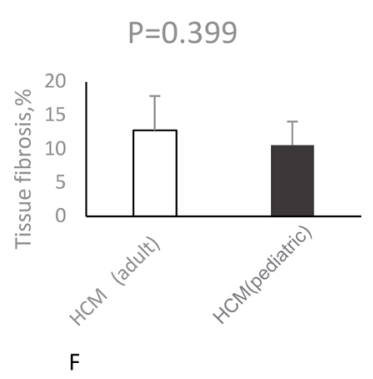

Figure 2. Changes in MVD and myocardial fibrosis area. MVD in pediatric patients (A), adult patients (B). Myocardial fibrosis area (\%) in pediatric patients $(C)$, adult patients (D). MVD changes in pediatric patients and adult patients $(E)$, and myocardial fibrosis area (\%) in pediatric patients and adult patients $(F)$.

\section{CONCLUSION}

This study concludes that pediatric patients and adult patients with HOCM present with significant myocardial fibrosis, suggesting the development of vascular endothelial cell lesions, reduced myocardial perfusion, myocardial interstitial remodeling, and microstructural fibrosis in childhood.

\section{REFERENCES}

Aguiar Rosa S, Rocha Lopes L, Fiarresga A, Ferreira RC, Mota Carmo M. 2021. Coronary microvascular dysfunction in hypertrophic cardiomyopathy: Pathophysiology, assessment, and clinical impact. Microcirculation. 28:e12656.

Antunes MO, Scudeler TL. 2020. Hypertrophic cardiomyopathy. Int J Cardiol Heart Vasc. 27:100503. 
Ariga R, Tunnicliffe EM, Manohar SG, et al. 2019. Identification of myocardial disarray in patients with hypertrophic cardiomyopathy and ventricular arrhythmias. J Am Coll Cardiol. 73:2493-02.

Avegliano G, Politi MT, Costabel JP, Kuschnir P, Trivi M, Ronderos R.2019. Differences in the extent of fibrosis in obstructive and nonobstructive hypertrophic cardiomyopathy. J Cardiovasc Med (Hagerstown). 20:389-96.

Chiribiri A, Leuzzi S, Conte MR, et al. 2015. Rest perfusion abnormalities in hypertrophic cardiomyopathy: correlation with myocardial fibrosis and risk factors for sudden cardiac death. Clin Radiol. 70:495-01.

Elliott PM, Anastasakis A, Borger MA, et al. 2014. 2014 ESC Guidelines on diagnosis and management of hypertrophic cardiomyopathy: the Task Force for the diagnosis and management of hypertrophic cardiomyopathy of the European society of cardiology. Eur Heart J. 35:2733-79.

Fernlund E, Gyllenhammar T, Jablonowski R, et al. 2017. Serum Biomarkers of Myocardial Remodeling and Coronary Dysfunction in Early Stages of Hypertrophic Cardiomyopathy in the Young. Pediatr Cardiol. 38:853-63.

Galati G, Leone O, Pasquale F, et al. 2016. Histological and histometric characterization of myocardial fibrosis in end-stage hypertrophic cardiomyopathy: A clinical-pathological study of 30 explanted hearts. Circ Heart Fail. 9:e003090.

Gersh BJ, Maron BJ, Bonow RO, et al. 2011.2011 ACCF/AHA guideline for the diagnosis and treatment of hypertrophic cardiomyopathy: a report of the American college of cardiology foundation/American heart association task force on practice guidelines. J Am Coll Cardiol. 58:e212-60.

Güçlü A, Happé C, Eren S, et al. 2015. Left ventricular outflow tract gradient is associated with reduced capillary density in hypertrophic cardiomyopathy irrespective of genotype. Eur J Clin Invest. 45:1252-9.

Ho CY, López B, Coelho-Filho OR, et al. 2010. Myocardial fibrosis as an early manifestation of hypertrophic cardiomyopathy. $\mathrm{N}$ Engl J Med.363:552-63.

Hughes SE. 2004. The pathology of hypertrophic cardiomyopathy. Histopathology. 44:412-27.

Iles LM, Ellims AH, Llewellyn H, et al. 2015. Histological validation of cardiac magnetic resonance analysis of regional and diffuse interstitial myocardial fibrosis. Eur Heart J Cardiovasc Imaging. 16:14-22.
Knaapen P, Germans T, Camici PG, et al. 2008. Determinants of coronary microvascular dysfunction in symptomatic hypertrophic cardiomyopathy. Am J Physiol Heart Circ Physiol. 294:H986-93.

Maron BJ. 2018. Clinical course and management of hypertrophic cardiomyopathy. N Engl J Med. 379:655-68.

Marszalek RJ, John Solaro R, Wolska BM. 2019. Coronary arterial vasculature in the pathophysiology of hypertrophic cardiomyopathy. Pflugers Arch. 471:769-80.

Matsunaka T, Hamada M, Matsumoto Y, Higaki J. 2003. First-pass myocardial perfusion defect and delayed contrast enhancement in hypertrophic cardiomyopathy assessed with MRI. Magn Reson Med Sci. 2:61-9.

Moravsky G, Ofek E, Rakowski H, et al. 2013. Myocardial fibrosis in hypertrophic cardiomyopathy: accurate reflection of histopathological findings by CMR. JACC Cardiovasc Imaging. 6:587-96.

Ostman-Smith I, Wettrell G, Keeton B, et al. 2008. Age- and genderspecific mortality rates in childhood hypertrophic cardiomyopathy. Eur Heart J. 29:1160-7.

Raman B, Ariga R, Spartera M, et al. 2019. Progression of myocardial fibrosis in hypertrophic cardiomyopathy: mechanisms and clinical implications. Eur Heart J Cardiovasc Imaging. 20:157-67.

Tangwiwat C, Kaolawanich Y, Krittayaphong R. 2019. Electrocardiographic predictors of myocardial fibrosis and apical hypertrophic cardiomyopathy. Ann Noninvasive Electrocardiol. 24: e12612.

Tian H, Yang C, Song Y, et al. 2018. Microvascular rarefaction and myocardial fibrosis in hypertrophic obstructive cardiomyopathy. Cardiology. $141: 202-11$

Timmer SA, Knaapen P. 2013. Coronary microvascular function, myocardial metabolism, and energetics in hypertrophic cardiomyopathy: insights from positron emission tomography. Eur Heart J Cardiovasc Imaging. 14:95-01.

Weissler-Snir A, Allan K, Cunningham K, et al. 2019. Hypertrophic cardiomyopathy-related sudden cardiac death in young people in Ontario. Circulation. 140:1706-16.

Ziolkowska L, Boruc A, Sobielarska-Lysiak D, et al. 2021. Prognostic significance of myocardial ischemia detected by single-photon emission computed tomography in children with hypertrophic cardiomyopathy. Pediatr Cardiol. 42:960-8. 